\title{
Generalized Epilepsy with Febrile Seizures Plus
}

National Cancer Institute

\section{Source}

National Cancer Institute. Generalized Epilepsy with Febrile Seizures Plus. NCI Thesaurus.

Code C122811.

A rare, genetically heterogeneous disorder caused by mutations in the SCN1A, GABRG2, GABRD, SCN9A, or STX1B genes. It is characterized by early childhood onset febrile seizures, generalized tonic-clonic seizures, absence seizures, myoclonic seizures, and atonic seizures 